IIIIIIIIIIIIIIIIII

\title{
Review
}

IIIIIIIIIIIIIIIIII

\section{Revisiting the Hansch-Fujita approach and development of a fundamental QSAR}

\author{
Seiji Hitaoka and Hiroshi Chuman* \\ Institution of Health Biosciences, The University of Tokushima Graduate School, 1-78 Shomachi, Tokushima 770-8505, Japan
}

(Received December 21, 2012; Accepted January 16, 2013)

\begin{abstract}
This review paper is based on the lecture presented in Kyoto on August 25th, 2012, by one of the authors (HC) in the Memorial Symposium for the 50th Anniversary of the Hansch-Fujita Approach; however, several parts have been added. The current paper contains (I) a formulation of an extended one of the well-known Hansch-Fujita QSAR analysis, but a new promising one (called linear expression by representative energy terms (LERE)-QSAR), which can consider ligand-protein interactions explicitly by using modern molecular calculations on a whole ligand-protein complex, and (II) applications of the LERE-QSAR analysis to several cases. () Pesticide Science Society of Japan

Keywords: ab initio molecular orbital calculation, Hansch-Fujita analysis, ligand-protein interaction, linear expression by representative energy terms (LERE), quantitative structure-activity relationship (QSAR), solvation energy calculation.
\end{abstract}

\section{Introduction}

The advent of QSAR began with the encounter of Professors Corwin Hansch and Toshio Fujita in June 1961, as described by themselves. ${ }^{1,2)}$ More than half a century has passed since their discovery of a general approach to the formulation of QSAR. Since its conception, this approach has provided a new perspective for chemical-biological interactions as well as a number of successes in drug discovery. Now it is time to develop a new and promising approach based on their QSAR with the aid of modern, powerful molecular calculations on a whole ligand-protein complex structure while staying true to the original ideas of Hansch and Fujita as much as possible. Their seminal ideas are succinctly and clearly expressed in their early publications. ${ }^{3-5)}$ With respect to their key concepts, which focused on (1) linear free-energy principle (LFEP) ${ }^{6}$ and additivity/decomposition of free-energy change and (2) LFEP parameters represented by Hammett $\sigma$, we now will discuss two topics relating to (1) and (2) by comparing results and implications obtained using the original and our QSAR approaches.

\section{From Hansch-Fujita Type of QSAR to a Novel One Using Molecular Calculations on Ligand-Protein Complexes}

\section{Hansch-Fujita type of QSAR}

A few remarks concerning the Hansch-Fujita equation may be

\footnotetext{
* To whom correspondence should be addressed.

E-mail: hchuman@tokushima-u.ac.jp

Published online April 7, 2013

(c) Pesticide Science Society of Japan
}

appropriate. A general form of the Hansch-Fujita equation is expressed by Eq. (1):

$$
\log (1 / C)=a \sigma+b \log P+\text { const }
$$

where $C$ is a concentration having a standard response in a standard time. $\sigma$ and $\log P$ are the Hammett constant and partition coefficient (to be more precise, logarithm of the partition coefficient $P$ between 1-octanol and water), respectively. When $C$ can be assumed to correspond to the equilibrium constant, $\log (1 / C)$ is proportional to the free-energy change $(\log (1 / C)=-\Delta G /$ $(2.303 R T))$ of the overall process from administration of a drug to interaction with a target receptor. Hence, Eq. (1) states that the overall free-energy change for a congeneric series of ligands is expressible as the sum of representative energy terms, each of which follows LFEP.

\section{Reassessment of parameters used in traditional QSAR, using molecular calculations \\ 2.1. Hammett $\sigma$}

The Hammett $\sigma$ constant has long been known as one of the most important LFEP parameters that correlate with biological activity. It is a conventionally used electronic parameter in studies of enzymatic QSAR. However, it is not necessarily obvious why $\sigma$ represents variations in the free-energy change associated with the complex formation between a congeneric series of ligands and their target protein. We comprehensively reevaluate experimentally derived parameter $\sigma$, confirming that it represents intermolecular interaction energy terms, by applying a molecular orbital (MO) calculation (Hartree-Fock (HF)/6$31 \mathrm{G}(\mathrm{d}, \mathrm{p}))$ to a simple ligand-protein complex model, complexes of a series of para and meta mono-substituted acetophenones 
with $N^{\prime}$-methylacetamide. ${ }^{7)}$ Figure 1 shows that the total binding energy $\left(\Delta E_{\mathrm{bind}}^{\mathrm{HF}}\right)$ is nicely linear with $\sigma$. Variations of energy components obtained with the energy decomposition analysis developed by Kitaura and Morokuma ${ }^{8)}$ are also plotted in Fig. 1 , revealing that all the energy components are linear with $\sigma$ and that the electrostatic component $(E S)$ governs $\Delta E_{\text {bind. }}^{\mathrm{HF}}$ Similarly, the hydration $\left(\Delta G_{\mathrm{sol}}\right)$ and dispersion $\left(E_{\text {disp }}\right)$ energy changes accompanying the complex formation are nicely linear with $\sigma$ ( $r=0.967$, slope $=-0.855$ (conductor-like polarizable continuum model $\left.(\mathrm{CPCM})^{9)} / \mathrm{HF} / 6-31 \mathrm{G}(\mathrm{d}, \mathrm{p})\right)$ and $r=0.973$, slope $=1.19$ $\left(\mathrm{MP} 2^{10)} / 6-31 \mathrm{G}(\mathrm{d}, \mathrm{p})\right)$, respectively). It is noteworthy that $\Delta G_{\mathrm{sol}}$ shows a nearly perfect correlation with $\Delta E_{\mathrm{bind}}^{\mathrm{HF}}(r=0.995$, slope $=$ -0.693 ). These results suggest that the intermolecular energy terms obtained through MO calculations for full complex structures of a congeneric series of ligands with a protein basically follow LFEP, as represented by Hammett $\sigma$, and can bridge the gap between the traditional Hansch-Fujita and modern QSAR approaches.

\subsection{Partition coefficient $\log P$}

The partition coefficient $\log P_{\text {sol }}\left(P_{\text {sol }}=C_{\text {sol }} / C_{\text {water }}\right)$ can be expressed with the Gibbs free-energy, enthalpy, and entropy differences.

$$
\begin{aligned}
\log P_{\text {sol }} & =-\Delta G_{\text {sol }} /(2.303 R T) \\
& =-\Delta H_{\text {sol }} /(2.303 R T)+\Delta S_{\text {sol }} /(2.303 R)
\end{aligned}
$$

Starting from the basic relation Eq. (2), we formulated a semiempirical correlation Eq. (3) for $\log P_{\text {sol }}$ (sol=chloroform

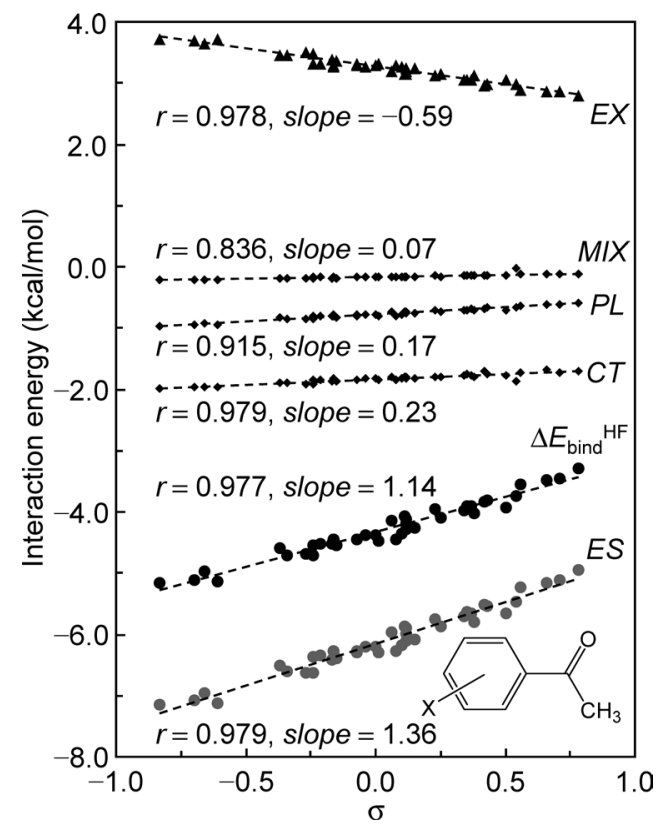

Fig. 1. Plot of Hammett $\sigma$ and energy components in complex formation of $N^{\prime}$-methylacetamide with 41 para and meta mono-substituted acetophenones (shown in the graph). $\Delta E_{\text {bind }}^{\mathrm{HF}}$ represents the total binding energy. $\Delta E_{\text {bind }}^{\mathrm{HF}}$ is further decomposed ${ }^{8)}$ into $E S$ (electrostatic), $E X$ (exchange), $C T$ (charge transfer), $P L$ (polarization), and $M I X$ (others) components $\left(\Delta E_{\mathrm{bind}}^{\mathrm{HF}}=E S+E X+C T+P L+M I X\right)$.
(CL) and 1-octanol (oct) $)^{11-13)}$ :

$$
\log P_{\mathrm{sol}}=a \Delta E_{\mathrm{sol}}+b A S A+c I_{\mathrm{HAc}}+d I_{\mathrm{sol}}+\mathrm{const}
$$

where $\Delta E_{\text {sol }}$ (calculated with CPCM/HF/6-31+G(d)//HF/3$\left.21 \mathrm{G}^{*}\right)$ is the solvation energy difference between aqueous and organic solvent phases, and ASA is the accessible surface area of solute molecules. $I_{\mathrm{HAc}}$ and $I_{\text {sol }}$ are indicator variables. $I_{\mathrm{HAc}}$ takes unity for hydrogen-bonding acceptors but otherwise zero, and $I_{\text {sol }}$ takes unity for $\log P_{\mathrm{CL}}$ and zero for $\log P_{\text {oct }}$. Although $I_{\mathrm{HAc}}$ and $I_{\text {sol }}$ were introduced empirically, physicochemical meanings of these indicator variables were discussed adequately in the original paper. ${ }^{12)}$ Equation (3) can give an excellent prediction of $\log P_{\text {sol }}$ for non-hydrogen bonders and hydrogen-bonding acceptors $(r=0.972, s=0.283$ for training compounds $(n=208)$ and $r=0.973, s=0.296$ for external test ones $(n=51))$. When deriving Eq. (3) from Eq. (2), we assumed that (1) $\Delta E_{\text {sol }}$ on the right-hand side of Eq. (3) corresponds to $\Delta H_{\text {sol }}$ in Eq. (2) (note that $\Delta H \approx \Delta E$ in solution) and that (2) $A S A, I_{\mathrm{HAc}}$, and $I_{\text {sol }}$ effectively express $\Delta S_{\text {sol }}$. In fact, the coefficient of $\Delta E_{\text {sol }}$ in Eq. (3) takes a value $(a=-0.776)$ close to the expected one $(-1 /(2.303 R T)=-0.734$ at $298 \mathrm{~K})$. However, for hydrogen-bonding donors, that is approximately half of the expected value $(\sim 0.34)$. This result probably suggests the deficiency in continuum solvation models (i.e., assumption of uniform distribution) used in PCM, ${ }^{9)}$ generalized Born (GB), ${ }^{14)}$ and Poisson-Boltzmann $(\mathrm{PB})^{14)}$ types of calculations. Figure 2 shows observed and calculated $\log P_{\mathrm{CL}}$ values (chloroform/water) of simple molecules, together with calculated ones with reference interaction site model (RISM), ${ }^{15,16)}$ which explicitly considers the (non-uniform) distribution of solvent molecules. The standard error of $\log P_{\mathrm{CL}}$ calculated with RISM (0.670) is, however, considerably larger than that with CPCM (0.296).

At present, it is difficult to reproduce $\log P_{\text {sol }}$ within chemical accuracy $(\sim 0.5 \mathrm{kcal} / \mathrm{mol})$ only by means of theoretical calculations such as the $a b$ initio self-consistent reaction field (SCRF) type of MOs and RISM. It should be very important to make the status of the current solvation models higher in predicting the solvation energy by extending and elaborating the current models.

\section{Formulation of the LERE-QSAR equation}

We previously proposed a novel QSAR procedure called linear expression by representative energy terms (LERE)-QSAR ${ }^{17-21 \text { ) }}$ involving molecular calculations such as an $a b$ initio fragment molecular orbital (FMO) one. ${ }^{22)}$ The idea of LERE-QSAR follows that of the Hansch-Fujita type of QSAR. The first assumption made in formulating the LERE-QSAR equation is that the free-energy terms comprising the overall free-energy change associated with complex formation are all additive.

$$
\Delta G_{\mathrm{obs}}=\Delta G_{\mathrm{bind}}+\Delta G_{\mathrm{sol}}+\Delta G_{\mathrm{others}}
$$

$\Delta G_{\text {obs }}$ on the left-hand side is the overall free-energy change calculated from the observed inhibitory potency $\left(\Delta G_{\mathrm{obs}}=R T \ln C\right.$, where $C$ is the same in Eq. (1)). $\Delta G_{\text {bind }}$ and $\Delta G_{\text {sol }}$ (typically taken 


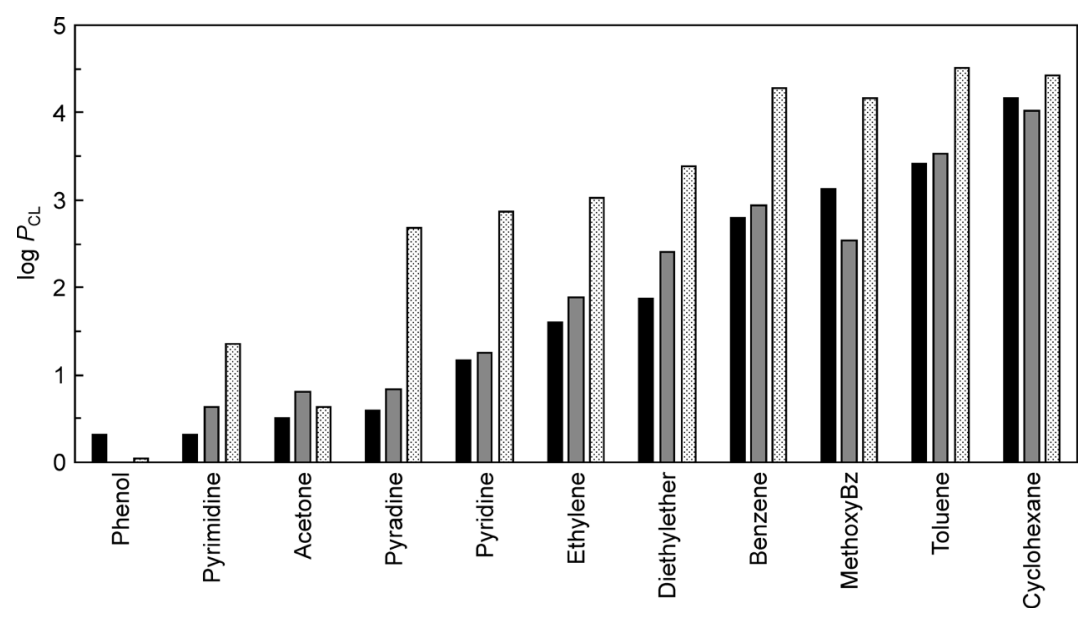

Fig. 2. Comparison of observed and calculated $\log P_{\mathrm{CL}}$ values (chloroform/water). Black, gray and dotted bars represent observed $\log P_{\mathrm{CL}}$ and calculated ones with $\mathrm{CPCM}^{12)}$ and RISM, ${ }^{15,16)}$ respectively.

(a)

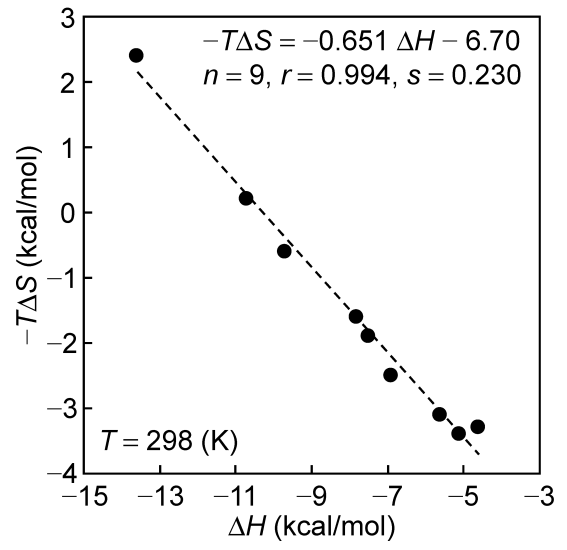

(b)

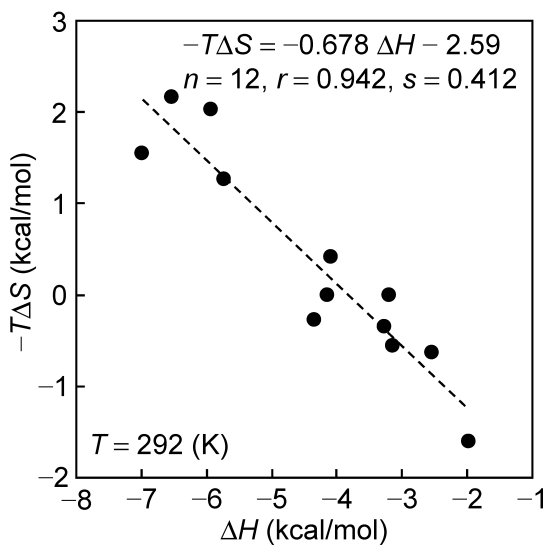

Fig. 3. Entropy-enthalpy compensation. (a) Human carbonic anhydrase II with a series of substituted benzenesulfonamides ${ }^{24)}$ and (b) concanavalin A, pea lectin, and lentil lectin with monosaccharides. ${ }^{25}$

as dominant free-energy terms) are the intrinsic binding interaction free-energy of an inhibitor with a protein and the solvation free-energy change associated with complex formation, respectively. $\Delta G_{\text {others }}$, the sum of free-energy terms other than representative free-energy terms $\Delta G_{\text {bind }}$ and $\Delta G_{\text {sol }}$, is assumed to be linear with that of representative free-energy terms (LERE approximation). $\Delta G_{\text {others }}$ consists of, for instance, the structural deformation (adaptation/induced fitting) energy associated with complex formation

$$
\Delta G_{\text {others }}=\beta\left(\Delta G_{\text {bind }}+\Delta G_{\text {sol }}\right)+\text { const }_{1}
$$

where $\Delta G_{\text {others }}$ is expected to work as a penalty term $(\beta<0$ and/ or const $\left.{ }_{1}>0\right)$. The last assumption is the entropy-enthalpy compensation, ${ }^{23)}$ which is expected to effectively hold for the binding of a series of ligands with a protein:

$$
T \Delta S=\alpha \Delta H+\text { const }_{2}
$$

where $\alpha>0$.

$\Delta G_{\text {sol }}$ is replaced by the dominant polar (electrostatic) contribution $\Delta G_{\text {sol }}^{\text {pol }}$ because the non-polar contribution is often negligible, and most of $\Delta G_{\text {sol }}^{\text {pol }}$ is considered to come from the enthalpic contribution. ${ }^{19)}$ Combining the above three equations yields the following expression (LERE-QSAR equation):

$$
\Delta G_{\mathrm{obs}}=\gamma\left(\Delta E_{\text {bind }}+\Delta G_{\text {sol }}^{\text {pol }}\right)+\text { const }_{3}
$$

The coefficient $\gamma$ on the right-hand side of the above equation is a constant determined by $\alpha$ and $\beta(\gamma=(1-\alpha)(1+\beta))$. Representative energy terms other than $\Delta E_{\text {bind }}$ and $\Delta G_{\text {sol }}^{\text {pol }}$, such as the dissociation of a ligand $\left(\Delta G_{\text {diss }}\right)$ and deformation free-energy changes, are added to Eq. (7) if needed. $\Delta E_{\text {bind }}$ is computable using $a b$ initio $\mathrm{MO}$ calculations such as $\mathrm{FMO}^{22)}$ and $\mathrm{ONIOM},{ }^{26)}$ and $\Delta G_{\text {sol }}^{\text {pol }}$ is computable with continuum solvation models such as $\mathrm{GB},{ }^{14}$ ) $\mathrm{PB},{ }^{14)} \mathrm{PCM},{ }^{9)}$ and RISM. ${ }^{27)}$ Table 1 concisely lists an estimation of representative energy terms and the entropic energy used in the LERE procedure. A general procedure for the LERE-QSAR analysis consists of two steps: (1) determine a complex structure, usually by carrying out classical molecular mechanics and molecular dynamics calculations repeatedly, and (2) calculate 
Table 1. Estimation of representative energy terms and the entropic energy

\begin{tabular}{cclc}
\hline Energy & Symbol & \multicolumn{1}{c}{ Method (program) } & Ref. \\
\hline Binding energy & \multirow{2}{*}{$\Delta E_{\text {bind }}$} & Molecular Mechanics (AMBER) & 28 \\
& & Fraction with Caps (MFCC) & 29 \\
& & FMO (ABINIT-MP and GAMESS) & 22 \\
& & QM/MM (ONIOM) & 26 \\
Solvation & \multirow{2}{*}{$\begin{array}{c}\text { energy } \\
\text { sol }\end{array}$} & GB/PB (AMBER) & 14 \\
& & PCM (Gaussian and GAMESS) & 9 \\
& & Hybrid (PCM/PB(GB)) & 30 \\
& & RISM & 27 \\
Entropic energy & $-T \Delta S$ & Normal mode calculation & 31,32 \\
& & Quasi-harmonic approximation & 33 \\
& & Entropy-enthalpy compensation & 23 \\
\hline
\end{tabular}

$\Delta X\left(X=E_{\text {bind }}\right.$ and $\left.G_{\text {sol }}^{\text {pol }}\right)$ as $\Delta X=X_{\text {complex }}-X_{\text {protein }}-X_{\text {ligand }}$ (supermolecular approximation).

Although several free-energy calculation methods ${ }^{34)}$ have been proposed such as the thermodynamics integration, freeenergy perturbation, Bennett's acceptance ratio, linear interaction energy (LIE), ${ }^{35)}$ and solvated interaction energy (SIE), ${ }^{36)}$ the purpose of our research is to develop an LFEP-based QSAR that can predict the overall binding free-energy change within chemical accuracy, which is unattainable by approaches other than LERE-QSAR, at least for now.

\section{Case Studies of LERE-QSAR Applications}

1. Comparative QSAR analysis of zinc containing enzyme inhibitors $^{18,20,21)}$

Substituted benzenesulfonamides (BSAs) and biphenylsulfonamides (BSs), shown in Figs. 4(a) and (b), inhibit bovine carbonic anhydrase II (CA) and matrix metalloproteinase-9 (MMP), respectively.

During their complex formation, the anionized sulfonamide $\left(\mathrm{SO}_{2} \mathrm{NH}^{-}\right)$in BSAs and carboxylate $\left(\mathrm{CO}_{2}^{-}\right)$in BSs coordinate to the zinc atom $\left(\mathrm{Zn}^{2+}\right)$ in the active sites of CA and MMP, respectively. Hansch ${ }^{37)}$ and Verma ${ }^{38)}$ reported QSAR Eqs. (8a) and (8b) for inhibition of CA by BSAs and that of MMP by BSs, respectively:

$$
\begin{gathered}
\log \left(1 / K_{\mathrm{i}}\right)=0.946 \sigma+0.177 \pi+5.52 \\
n=16, \quad r=0.956, \quad s=0.193 \\
\log \left(1 / \mathrm{IC}_{50}\right)=-1.14 \sigma+1.24 M R+4.38 \\
n=9, \quad r=0.944, \quad s=0.195
\end{gathered}
$$

where $\pi$ in Eq. (8a) is a hydrophobic constant of a substituent ( $X$ in Fig. 4(a)), which is defined as $\log P$ (substituted benzene) $-\log P$ (benzene). $M R$ in Eq. (8b) represents the dispersion interaction of a substituent ( $X$ in Fig. $4(\mathrm{~b})$ ). It should be noted that signs of coefficient of $\sigma$ are opposite in Eqs. (8a) and (8b); thus, we focused on the reasons the opposite direction of substituent electronic effect is observed in complex formations CA-BSA and MMP-BS. We performed the LERE-QSAR (a)

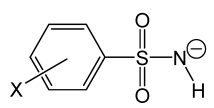

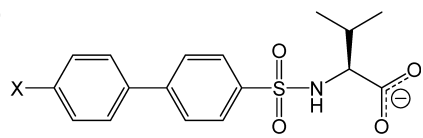

Fig. 4. Structure of (a) BSAs (para- and meta-substituted benzenesulfonamides) and (b) BSs (para-substituted biphenylsulfonamides).

analysis using the three representative energy terms: $\Delta E_{\text {bind }}$ (ONIOM (HF-D ${ }^{39} / 6-31 \mathrm{G}$ : Amber), electronic embedding scheme), $\Delta G_{\text {sol }}^{\text {pol }}(\mathrm{GB})$, and $\Delta G_{\text {diss }}$ (integral equation formalism PCM (IEFPCM ${ }^{40)} / \mathrm{B}^{2} \mathrm{LYP}^{41)} / 6-31+\mathrm{G}(\mathrm{d}, \mathrm{p})$ and IEFPCM/HF/6$31+\mathrm{G}(\mathrm{d})$ for CA-BSAs ${ }^{18,20)}$ and $\mathrm{MMP}-\mathrm{BSs},{ }^{21)}$ respectively) for complexes CA-BSA and MMP-BS and obtained Eqs. (9a) and (9b) with $s$ (standard deviation) $<0.3 \mathrm{kcal} / \mathrm{mol}$ :

$$
\begin{aligned}
& \Delta G_{\text {obs }}=\gamma\left(\Delta E_{\text {bind }}+\Delta G_{\text {sol }}^{\mathrm{pol}}\right)+0.337 \Delta G_{\text {diss }}+2.14 \\
& n=16, \quad r=0.952, \quad s=0.268 \mathrm{kcal} / \mathrm{mol}, \quad \gamma=0.205 \\
& \Delta G_{\text {obs }}=\gamma\left(\Delta E_{\text {bind }}+\Delta G_{\text {sol }}^{\text {pol }}\right)-1.56 \Delta G_{\text {diss }}+29.5 \\
& n=9, \quad r=0.957, \quad s=0.234 \mathrm{kcal} / \mathrm{mol}, \quad \gamma=0.231
\end{aligned}
$$

It is noted that the coefficients $(\gamma)$ in Eqs. (9a) and (9b) are nearly the same value. Figure 5(a) shows that the relative contribution from $\Delta G_{\text {sol }}^{\text {pol }}$ and $\Delta G_{\text {diss }}$ overwhelms that from $\Delta E_{\text {bind }}$ and that the former governs the overall free-energy change $\Delta G_{\text {obs }}$. Conversely, Fig. 5(b) shows that the latter governs $\Delta G_{\text {obs. }}$ There is an anticorrelation between $\Delta E_{\text {bind }}$ and $\Delta G_{\text {sol }}^{\text {pol }}$ in Eqs. (9a) and (9b) ( $r=-0.922$ and -0.862 , respectively (the negative sign of $r$ indicates a negative slope hereafter)), as frequently observed. As demonstrated in the previous section "Hammett $\sigma$," $\Delta E_{\text {bind }}$ and $\Delta G_{\text {sol }}^{\text {pol }}$ are expected to show positive and negative correlations with Hammett $\sigma$, respectively.

As can be seen in Figs. 6(a) and (b), variations of $\Delta G_{\text {sol }}^{\text {pol }}$ in CA-BSAs and $\Delta E_{\text {bind }}$ in MMP-BSs are mostly from the zinc atom.

The opposite signs of the coefficients of $\sigma$ in Eqs. (8a) and (8b) are now clearly explainable from differences in representative energy terms between complex formations CA-BSA and MMP-BS. This instructive case study demonstrates that LEREQSAR has a considerable advantage over the traditional QSAR, although there is no logical conflict between the two.

2. Comparative LERE-QSAR analysis of inhibitory activity of sialic acid analogues on two neuraminidases ${ }^{17,19)}$

Neuraminidases (NAs) are ubiquitous exoglycohydrolases that hydrolyze the terminal sialic acids of glycoproteins and glycolipids and are widely distributed in nature, from viruses, microorganisms, and fungi to higher animals and humans. ${ }^{42)}$ Among them, influenza virus NAs have been studied extensively as a target for influenza disease, and anti-influenza drugs have been developed. These drugs have the potential to affect the activities of endogenous human NAs. ${ }^{43,44)}$ Thus, it is of great importance to examine differences in the binding mechanism between the influenza virus and human NAs with a series of sialic acid analogues. We selected influenza virus neuraminidase-1 (N1-NA) 
(a)

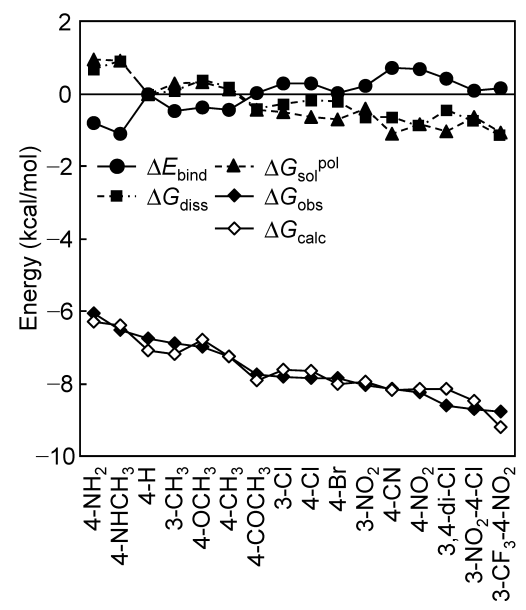

(b)

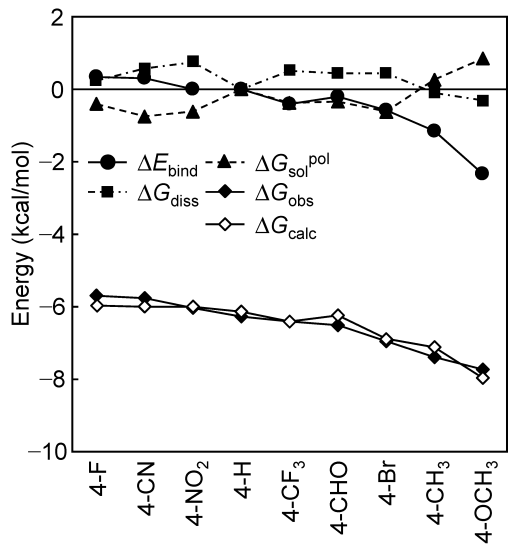

Fig. 5. Variation of energy components. (a) CA-BSAs and (b) MMP-BSs.

(a)

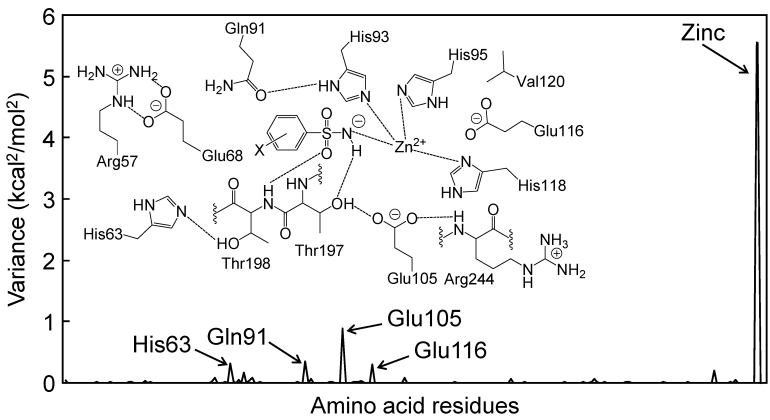

(b)

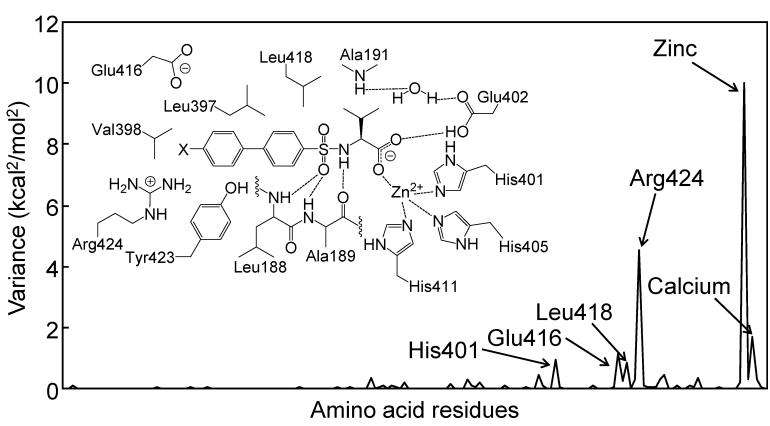

Fig. 6. Variance of (a) $\Delta G_{\text {sol }}^{\text {pol }}$ of CA-BSAs and (b) $\Delta E_{\text {bind }}$ of MMPBSs, together with interaction schemes of (a) and (b). $\Delta G_{\text {sol }}^{\text {pol }}$ and $\Delta E_{\text {bind }}$ are calculated with GB and molecular mechanics (AMBER), respectively. The variance of quantities $\left(X_{i}, i=1, n\right)$ is defined as $\Sigma\left(X_{i}-\langle X\rangle\right)^{2}$, where $\langle X\rangle=(1 / n) \Sigma X_{i}$.

and human neuraminidase-2 (hNEU2). Amino acid residues important for recognition of sialic acids are basically conserved in N1-NA and hNEU2. ${ }^{19}$

Table 2 lists sialic acid analogues, Sets I and II compounds used in the study. We constructed complexes of N1-NA and hNEU2 with Sets I and II compounds, respectively, and then performed the LERE-QSAR analysis using the three representative energy terms: $\Delta E_{\text {bind }}\left(=\Delta E_{\text {bind }}^{\mathrm{HF}}+E_{\text {disp }}, \mathrm{FMO} / \mathrm{MP} 2 / 6-31 \mathrm{G}\right)$,
$\Delta G_{\text {sol }}^{\text {pol }}$ (hybrid $(\mathrm{CPCM} / \mathrm{PB})^{30)}$ and $\mathrm{PB}$ for N1-NA-Set I and hNEU2-Set II compounds, respectively), and $\Delta G_{\text {diss }}$ (CPCM/ $\mathrm{HF} / 6-31+\mathrm{G}(\mathrm{d}, \mathrm{p}))$.

The observed overall free-energy change $\Delta G_{\text {obs }}$ for complex formation of N1-NA and hNEU2 with sialic acid analogues is excellently reproducible with the LERE-QSAR Eqs. (10a) and (10b), respectively $(s<0.4 \mathrm{kcal} / \mathrm{mol})$.

$$
\begin{gathered}
\Delta G_{\mathrm{obs}}=\gamma\left(\Delta E_{\mathrm{bind}}^{\mathrm{HF}}+E_{\mathrm{disp}}+\Delta G_{\text {sol }}^{\mathrm{pol}}\right)+0.0708 \Delta G_{\mathrm{diss}}+25.4 \quad(10 \mathrm{a}) \\
n=8, \quad r=0.988, \quad s=0.389 \mathrm{kcal} / \mathrm{mol}, \quad \gamma=0.561 \\
\Delta G_{\mathrm{obs}}=\gamma\left(\Delta E_{\mathrm{bind}}^{\mathrm{HF}}+E_{\mathrm{disp}}+\Delta G_{\mathrm{sol}}^{\mathrm{pol}}\right)+0.0976 \Delta G_{\mathrm{diss}}-2.25 \quad(10 \mathrm{~b}) \\
n=7, \quad r=0.985, \quad s=0.237 \mathrm{kcal} / \mathrm{mol}, \quad \gamma=0.0476
\end{gathered}
$$

As can be seen in Figs. $7(\mathrm{a})$ and (b), $\Delta G_{\text {diss }}$ varies depending on the types of cationic groups in fragment $\mathbf{C}\left(\mathrm{NH}_{3}^{+}\right.$and $\mathrm{NHC}\left(=\mathrm{NH}_{2}^{+}\right) \mathrm{NH}_{2}$, shown in Table 2). Figure 7(a) for N1-NASet I compounds shows that the electrostatic contribution from two electrostatic energy terms ( $\Delta E_{\mathrm{bind}}^{\mathrm{HF}}$ and $\left.\Delta G_{\mathrm{sol}}^{\mathrm{pol}}\right)$ in Eq. (10a) is considerably smaller than that from the dispersion energy term $\left(E_{\text {disp }}\right)$ because of compensation between the two electrostatic energy terms $(r=-0.950)$. Consequently, $E_{\text {disp }}$ governs $\Delta G_{\text {obs }}$ in complex formation of N1-NA with Set I compounds. ${ }^{17)}$ Conversely, Fig. 7(b) for hNEU2-Set II compounds shows that the contribution of $E_{\text {disp }}$ is considerably smaller than that of the electrostatic energy contribution in Eq. (10b). Hence, $\Delta E_{\text {bind }}^{\mathrm{HF}}$ governs $\Delta G_{\text {obs }}$ in complex formation of hNEU2 with Set II compounds. ${ }^{19)}$

It should be noted that remarkable differences in the coefficient $(\gamma)$ and intercept (corresponds to const $_{3}$ in Eq. (7)) exist between Eqs. (10a) and (10b). The coefficient $(\beta)$ and intercept (const ${ }_{1}$ ) in Eq. (5) (LERE approximation) are expressed with $\alpha, \gamma$, const $_{2}$, and const $_{3}$ in Eqs. (6) and (7): $(\alpha+\gamma-1) /(1-\alpha)$ and $\left(\right.$ const $_{2}+$ const $\left._{3}\right) /(1-\alpha)$, respectively. When assuming $\alpha=$ $0.70,{ }^{25,47-49)} \beta$ takes 0.87 and -0.84 , and const $_{3}$ is $85+3.3$ const $_{2}$ (relative value: $85 \mathrm{kcal} / \mathrm{mol})$ and $-7.5+3.3$ const $_{2}(-7.5 \mathrm{kcal} /$ mol) for Eqs. (10a) and (10b), respectively. Using $\beta$ and const $_{1}$ 
Table 2. Chemical structure and inhibitory potency of Sets I and II compounds toward N1-NA and hNEU2

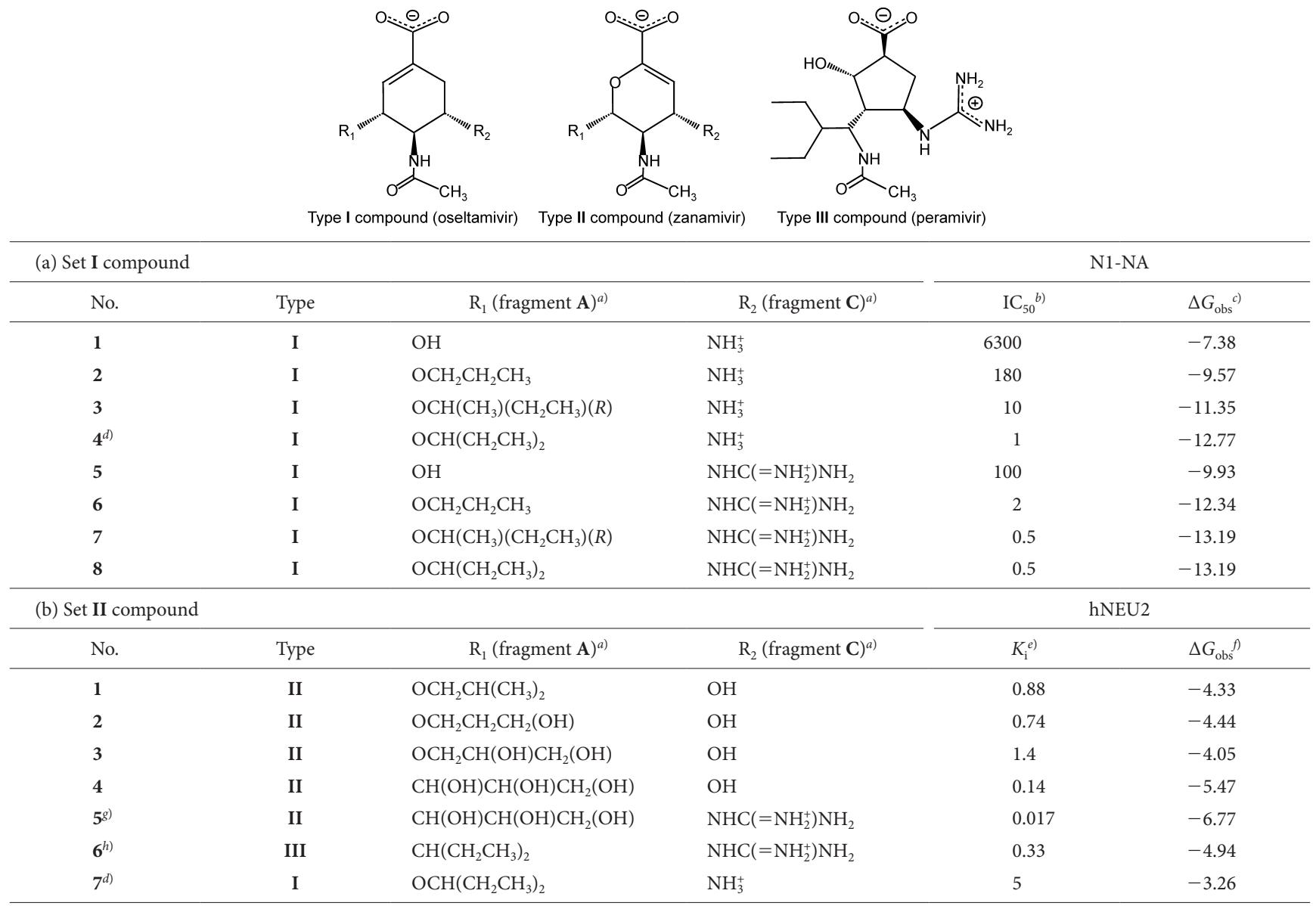

a) Fragments $\mathbf{A}$ and $\mathbf{C}$ correspond to $\mathrm{R}_{1}$ and $\mathrm{R}_{2}$, respectively. Fragment $\mathbf{B}$ denotes the rest of structure (without fragments $\mathbf{A}$ and $\mathbf{C}$ ). ${ }^{b)}$ Taken from Ref. 45 (In $\mathrm{nM}){ }^{c}{ }^{c} \Delta G_{\mathrm{obs}}=R T \ln \mathrm{IC}_{50}(T=310 \mathrm{~K}) .{ }^{d)}$ Oseltamivir (Tamiflu). ${ }^{e}$ Taken from Ref. 46 (In mM). ${ }^{. f} \Delta G_{\mathrm{obs}}=R T \ln K_{\mathrm{i}}(T=310 \mathrm{~K}) .{ }^{g}$ Zanamivir (Relenza). ${ }^{h}$ Peramivir (Rapiacta).

(a)

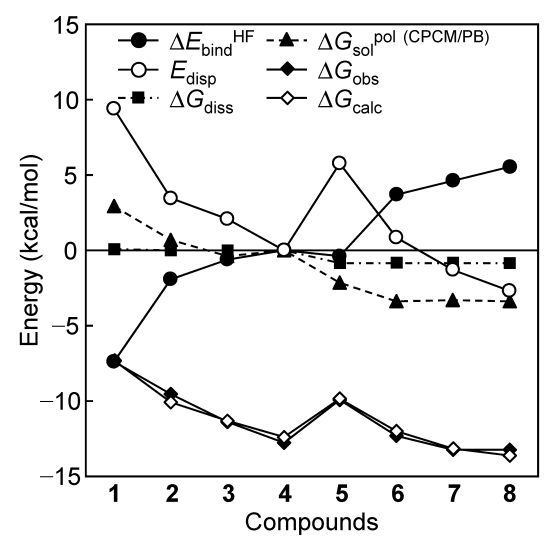

(b)

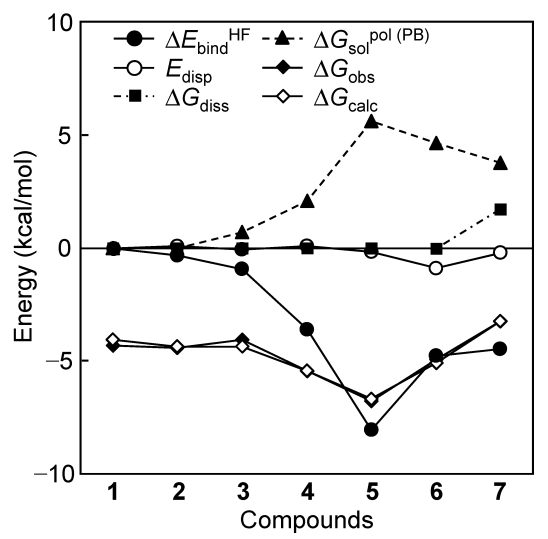

Fig. 7. Variation of energy components. (a) N1-NA and (b) hNEU2.

in Eq. (5) (LERE approximation/assumption), we will now discuss the effect of $\Delta G_{\text {others }}$ on the coefficient and intercept in Eqs. (10a) and (10b). The positive $\beta(0.87)$ and large const ${ }_{1}(85 \mathrm{kcal} /$ mol) for Eq. (10a) indicate that $\Delta G_{\text {others }}$ varies in a parallel manner with the sum of representative energy terms and yields a large positive constant in Eq. (10a) as a penalty energy. In contrast, the negative $\beta(-0.84)$ and smaller const ${ }_{1}(-7.5 \mathrm{kcal} / \mathrm{mol})$ for Eq. (10b) indicate that $\Delta G_{\text {others }}$ nearly cancels out the sum of representative energy terms because of negative correlation with the sum. As expected ( $\beta<0$ and/or const $\left.{ }_{1}>0\right), \Delta G_{\text {others }}$ is 
(a)

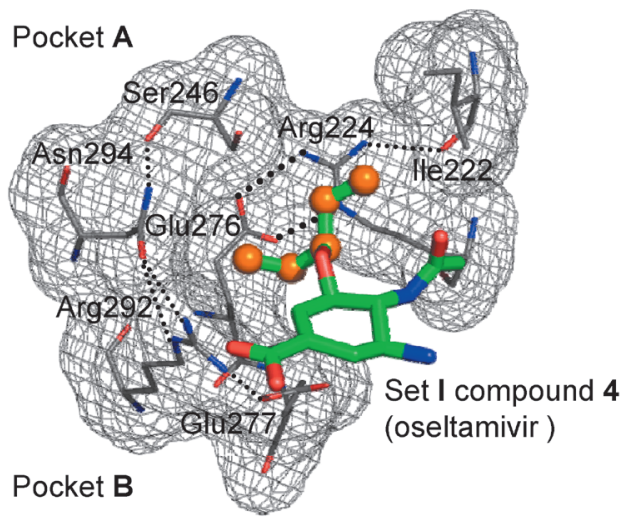

(c)

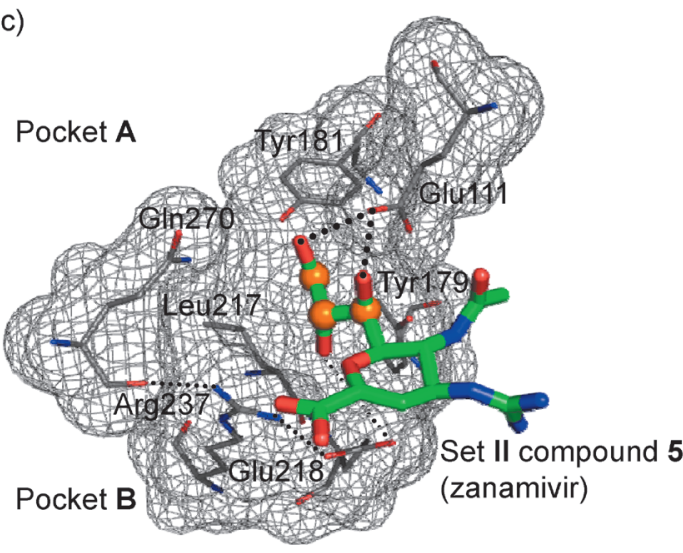

(b)

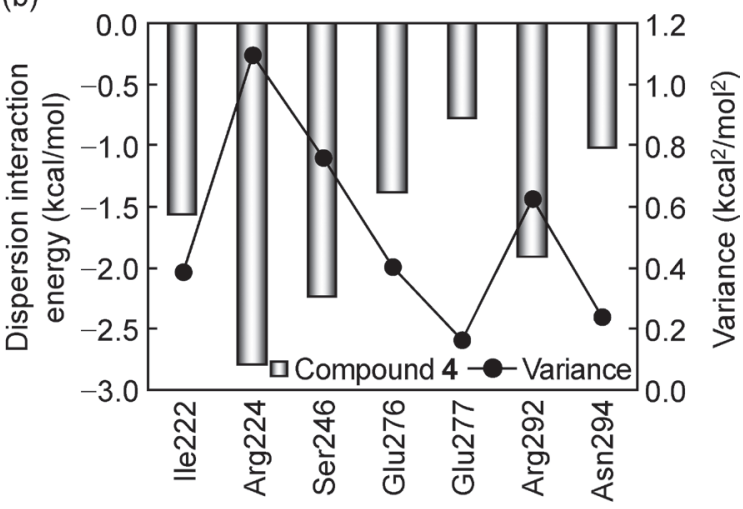

(d)

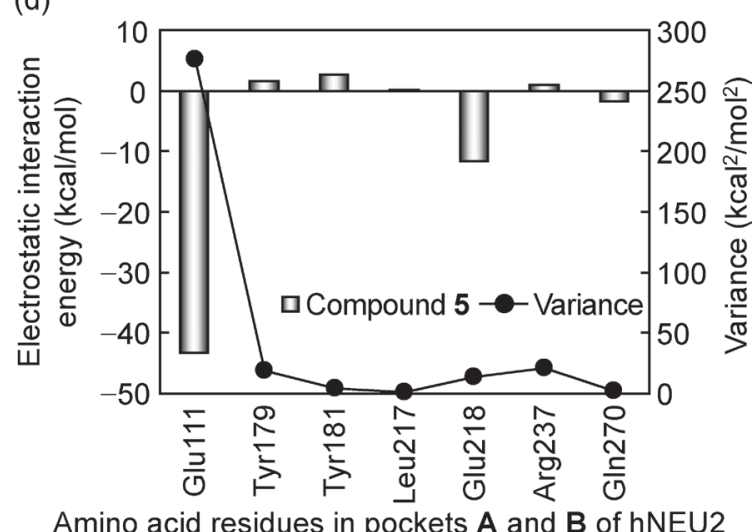

Amino acid residues in pockets $A$ and $B$ of hNEU2

Fig. 8. (a) Set I compound 4 (oseltamivir) accommodated in the active site of N1-NA. (b) Dispersion interaction energies of fragment A in Set I compound 4 with amino acid residues in pockets $\mathbf{A}$ and $\mathbf{B}$ of N1-NA. Pockets $\mathbf{A}$ and $\mathbf{B}$ correspond to amino acid residues surrounding fragments $\mathbf{A}$ and $\mathbf{B}$, respectively. (c) Set II compound $\mathbf{5}$ (zanamivir) accommodated in the active site of hNEU2. (d) Electrostatic interaction energies of fragment A in Set II compound $\mathbf{5}$ with amino acid residues in pockets $\mathbf{A}$ and $\mathbf{B}$ of hNEU2.

now confirmed to work as the penalty energy both in Eqs. (10a) and (10b) but behaves differently. This is probably due to differences in the primary interaction governing the overall free-energy change, $E_{\text {disp }}$ and $\Delta E_{\text {bind }}^{\mathrm{HF}}$ for N1-NA-Set I and hNEU2-Set II compounds, respectively. Figures $8(\mathrm{a}),(\mathrm{b})$ and (c), (d) show that contributions of $E_{\text {disp }}$ (N1-NA-Set I compounds) and $\Delta E_{\text {bind }}^{\mathrm{HF}}$ (hNEU2-Set II compounds) from amino acid residues located in close proximity to fragment $\mathbf{A}$ (shown in Table 2) dominantly determine the overall free-energy change $\Delta G_{\text {obs }}$, respectively.

The second case study demonstrates that the LERE-QSAR equation is significantly informative concerning variations of ligand-protein interaction energy.

\section{Concluding Remarks}

We feel some concern about QSAR models in which descriptors (explaining variables) are selected "automatically" from a large pool of candidate descriptors, as sometimes found in recent publications. We believe QSAR models should be interpretable physicochemically and informative chemically and biologically, not merely statistically acceptable. It is important to make efforts for constructing a fundamental QSAR that can accurately describe the essence of chemical-biological interactions. As mentioned at the beginning of this paper, the encounter of the two perspicacious scientists has had a strong and lasting influence on later generations.

\section{Acknowledgments}

We would like to express our sincere gratitude to Professor Toshio Fujita (Kyoto University), who has been giving us valuable and warm suggestions during the course of our studies. We also sincerely thank Professor Cynthia Selassie (Pomona College, US) for improving words and $\mathrm{Mr}$. Takuya Sugimoto (University of Tokushima) for preparing a part of figures.

\section{References}

1) C. Hansch: J. Comput. Aided Mol. Des. 25, 495-507 (2011).

2) T. Fujita: J. Comput. Aided Mol. Des. 25, 509-517 (2011).

3) C. Hansch and T. Fujita: J. Am. Chem. Soc. 86, 1616-1626 (1964).

4) T. Fujita, J. Iwasa and C. Hansch: J. Am. Chem. Soc. 86, 5175-5180 (1964).

5) T. Fujita and T. Ban: J. Med. Chem. 14, 148-152 (1971).

6) L. P. Hammett: "Quantitative Relationships Involving Structure and Reactivity," in Physical Organic Chemistry (Chapter 11), Second ed., McGraw-Hill Kogakusha Ltd., Tokyo, pp. 347-390, 1970.

7) T. Yoshida, M. Shimizu, M. Harada, S. Hitaoka and H. Chuman: 
Bioorg. Med. Chem. Lett. 22, 124-128 (2012).

8) K. Kitaura and K. Morokuma: Int. J. Quantum Chem. 10, 325-340 (1976).

9) A. Klamt: J. Phys. Chem. 99, 2224-2235 (1995).

10) M. Head-Gordon, J. A. Pople and M. J. Frisch: Chem. Phys. Lett. 153, 503-506 (1988).

11) H. Chuman, A. Mori and H. Tanaka: Anal. Sci. 18, 1015-1020 (2002).

12) H. Chuman, A. Mori, H. Tanaka, C. Yamagami and T. Fujita: J. Pharm. Sci. 93, 2681-2697 (2004).

13) H. Chuman: SAR QSAR Environ. Res. 19, 71-79 (2008).

14) P. A. Kollman, I. Massova, C. Reyes, B. Kuhn, S. Huo, L. Chong, M. Lee, T. Lee, Y. Duan, W. Wang, O. Donini, P. Cieplak, J. Srinivasan, D. A. Case and T. E. Cheatham 3rd: Acc. Chem. Res. 33, 889-897 (2000).

15) K. Sato, H. Chuman and S. Ten-no: J. Phys. Chem. B 109, 1729017295 (2005).

16) S. Ten-no, J. Jung, H. Chuman and Y. Kawashima: Mol. Phys. 108, 327-332 (2010).

17) S. Hitaoka, M. Harada, T. Yoshida and H. Chuman: J. Chem. Inf. Model. 50, 1796-1805 (2010).

18) T. Yoshida, Y. Munei, S. Hitaoka and H. Chuman: J. Chem. Inf. Model. 50, 850-860 (2010).

19) S. Hitaoka, H. Matoba, M. Harada, T. Yoshida, D. Tsuji, T. Hirokawa, K. Itoh and H. Chuman: J. Chem. Inf. Model. 51, 2706-2716 (2011).

20) Y. Munei, K. Shimamoto, M. Harada, T. Yoshida and H. Chuman: Bioorg. Med. Chem. Lett. 21, 141-144 (2011).

21) T. Yoshida, S. Hitaoka, A. Mashima, T. Sugimoto, H. Matoba and H. Chuman: J. Phys. Chem. B 116, 10283-10289 (2012).

22) K. Kitaura, E. Ikeo, T. Asada, T. Nakano and M. Uebayasi: Chem. Phys. Lett. 313, 701-706 (1999).

23) E. Freire: Chem. Biol. Drug Des. 74, 468-472 (2009).

24) A. D. Scott, C. Phillips, A. Alex, M. Flocco, A. Bent, A. Randall, R. O'Brien, L. Damian and L. H. Jones: ChemMedChem 4, 1985-1989 (2009).

25) F. P. Schwarz, K. D. Puri, R. G. Bhat and A. Surolia: J. Biol. Chem. 268, 7668-7677 (1993).

26) F. Maseras and K. Morokuma: J. Comput. Chem. 16, 1170-1179 (1995).

27) D. Chandler and H. C. Andersen: J. Chem. Phys. 57, 1930-1937 (1972).

28) S. J. Weiner, P. A. Kollman, D. A. Case, U. C. Singh, C. Ghio, G. Alagona, S. Profeta and P. Weiner: J. Am. Chem. Soc. 106, 765-784 (1984).

29) D. W. Zhang, X. H. Chen and J. Z. H. Zhang: J. Comput. Chem. 24, 1846-1852 (2003).
30) S. Hitaoka, H. Matoba, A. Kawano, Y. Shibata, M. Harada, S. Sakamoto, T. Yoshida and H. Chuman: Abstr. 40th Symposium on StructureActivity Relationships, pp. 86-89, 2012 (in Japanese).

31) N. Go, T. Noguti and T. Nishikawa: Proc. Natl. Acad. Sci. U.S.A. 80, 3696-3700 (1983).

32) B. Brooks and M. Karplus: Proc. Natl. Acad. Sci. U.S.A. 80, 6571-6575 (1983).

33) J. Schlitter: Chem. Phys. Lett. 215, 617-621 (1993).

34) J. Wereszczynski and J. A. McCammon: Q. Rev. Biophys. 45, 1-25 (2012).

35) J. Åqvist C. Medina and J. E. Samuelsson: Protein Eng. 7, 385-391 (1994).

36) M. Naïm, S. Bhat, K. N. Rankin, S. Dennis, S. F. Chowdhury, I. Siddiqi, P. Drabik, T. Sulea, C. I. Bayly, A. Jakalian and E. O. Purisima: J. Chem. Inf. Model. 47, 122-133 (2007).

37) C. Hansch: Farmaco 58, 625-629 (2003).

38) R. P. Verma and C. Hansch: Bioorg. Med. Chem. 15, 2223-2268 (2007).

39) S. J. Grimme: J. Comput. Chem. 27, 1787-1799 (2006).

40) E. Cancès, B. Mennucci and J. Tomasi: J. Chem. Phys. 107, 3032-3041 (1997).

41) A. D. Becke: J. Chem. Phys. 98, 5648-5652 (1993).

42) K. E. Achyuthan and A. M. Achyuthan: Comp. Biochem. Physiol. B Biochem. Mol. Biol. 129, 29-64 (2001).

43) K. Hata, K. Koseki, K. Yamaguchi, S. Moriya, Y. Suzuki, S. Yingsakmongkon, G. Hirai, M. Sodeoka, M. von Itzstein and T. Miyagi: Antimicrob. Agents Chemother. 52, 3484-3491 (2008).

44) C.-Y. Li, Q. Yu, Z.-Q. Ye, Y. Sun, Q. He, X.-M. Li, W. Zhang, J. Luo, X. Gu, X. Zheng and L. Wei: Cell Res. 17, 357-362 (2007).

45) C. U. Kim, W. Lew, M. A. Williams, H. Wu, L. Zhang, X. Chen, P. A. Escarpe, D. B. Mendel, W. G. Laver and R. C. Stevens: J. Med. Chem. 41, 2451-2460 (1998).

46) L. M. G. Chavas, R. Kato, N. Suzuki, M. von Itzstein, M. C. Mann, R. J. Thomson, J. C. Dyason, J. McKimm-Breschkin, P. Fusi, C. Tringali, B. Venerando, G. Tettamanti, E. Monti and S. Wakatsuki: J. Med. Chem. 53, 2998-3002 (2010).

47) D. Gupta, M. Cho, R. D. Cummings and C. F. Brewer: Biochemistry 35, 15236-15243 (1996).

48) C. P. Swaminathan, N. Surolia and A. Surolia: J. Am. Chem. Soc. 120, 5153-5159 (1998).

49) T. M. Gloster, P. Meloncelli, R. V. Stick, D. Zechel, A. Vasella and G. J. Davies: J. Am. Chem. Soc. 129, 2345-2354 (2007). 